\title{
Flow-Induced Crystallization of PB-1: From the Low Shear Rate Region up to Processing Rates
}

\author{
Jimmy Baert and Peter Van Puyvelde* \\ Department of Chemical Engineering, Katholieke Universiteit Leuven, W. De Croylaan 46, B-3001, \\ Leuven, Belgium

\section{Florentin Langouche*} \\ Solvay Central Laboratory, Neder Over Heembeek, Rue de Ransbeek 310, B-1120, Brussels, Belgium \\ Received September 6, 2006; Revised Manuscript Received October 5, 2006
}

\begin{abstract}
In this work a global view of flow-induced orientation in isotactic PB-1 is presented. The shear cell used in this work submits the sample to a homogeneous flow field and can be operated from the low shear rate region up into the highly oriented regime close to industrial processing conditions. For varying levels of shear rate and shear strain and for several crystallization temperatures, the transmitted intensity and birefringence are measured throughout the complete crystallization process and characteristic crystallization times are defined. Different regimes of flow-induced crystallization are observed as a function of an increased level of molecular orientation and stretch. Results concerning the Fase II to Fase I transformation in PB-1 are also presented.
\end{abstract}

\section{Introduction}

The reason for the intense interest in crystallization of flowing polymer melts, when even quiescent crystallization is not yet completely understood, is its immense technological relevance to polymer processing. Semicrystalline polymers comprise nearly two-thirds of all synthetic polymers. These are processed to form films, fibers, and moulded articles using operations such as extrusion, moulding, fiber spinning, film blowing, etc. During this processing, the polymer melt is subjected to complex and intense flow fields (shear, elongational, or mixed) after which the polymer crystallizes. The morphology of the semicrystalline polymer in the final product, and subsequently its properties and quality, depend on the manner in which the polymer crystallizes from the flowing melt.

In the last few decades, there have been several investigations into the route by which a polymer melt, subjected to flow and a certain degree of undercooling, transforms into a crystalline state (see for instance a recent review by Kumaraswamy ${ }^{1}$ ). The renewed interest in this problem is due to the availability of new experimental tools that, coupled with advances in modeling and simulations, are beginning to yield insights into the molecular characteristics that control the crystallization pathways adopted by a stressed polymer melt. Most of the studies have concentrated on shear flow, which will also be the focus of this paper.

It is now well-known that applying shear flow to an undercooled polymer melt enhances the crystallization process, mainly through an increase in nucleation. ${ }^{2-12}$ The extent of this enhancement depends on many factors, such as the processing parameters and the molecular properties of the polymer. Despite numerous studies, the picture on crystallization in quiescent and sheared conditions is far from complete. To further improve our understanding of the crystallization process from a fundamental point of view, it has been proven useful to separate the thermal and flow effects. The "short-time shearing" protocol, developed by Janeschitz-Kriegl and co-workers, ${ }^{13}$ enables

* To whom correspondence should be addressed. E-mail: Peter.Vanpuyvelde@cit.kuleuven.be (P.V.P.); Florentin.Langouche@ solvay.com (F.L.) separation of flow, nucleation, and growth phenomena. Since its introduction, it has been widely used to study flow-induced crystallization. Many of the reported results have been obtained in the low shear rate region, using either rheometrical equipment ${ }^{14-16}$ or using commercial shear cells such as for instance the Linkam CSS-450.,17-20 Experimental results extending into the high shear rate region, and hence close to industrial processing rates, are much more limited. The research group of Janeschitz-Kriegl 13,21-24 used their "short-time shearing" protocol in combination with an extrusion die to study the effects of intense shear flow. The experimental approach of Kumaraswamy et al. 2,25,26 used the same setup and protocol combined with multiple probes of structure including turbidity, polarimetry, small-angle X-ray scattering, wide-angle X-ray scattering, and optical and electron microscopy to investigate the development of structure and the time scale over which this microstructure originates. A major drawback of extrusion die setups is the nonhomogeneous character of the flow field: the velocity gradient is much higher at the die walls than in the center, resulting in a shear rate that is not constant throughout the sample. This makes it more difficult to ascribe the various layers exactly to certain shear rates and shearing times. Moreover, the optical signals are indicative mainly of structure formation near the die walls, since the polymer near the wall crystallizes before the region near the center as a result of experiencing a higher shear rate and strain. In the present paper flow-induced crystallization of two PB-1s with a different molecular weight and a similar polydispersity is studied using a newly developed sandwich-type shear cell $^{27}$ that can be operated from the low shear rate region up to rates that generate a highly oriented structure. The advantage of this shear cell is that it imposes a homogeneous shear flow resulting in a constant shear rate throughout the entire sample. Hence the optical signals are not dominated by structure formation near the walls. The influence of the degree of undercooling and the magnitude of shear flow will be the main parameters studied.

PB-1 displays relatively slow crystallization kinetics at low to moderate degrees of undercooling, allowing a large time window for observation. Recent publications ${ }^{3,12,28-30}$ are also 
concerned with the flow-induced crystallization of PB-1 and attempts have been made to reconcile experimental results and crystallization theory by the use of microrheological modeling 28 and scaling laws for characteristic crystallization times as a function of molecular parameters and processing conditions. ${ }^{3,12,30}$ These studies are restricted to relatively low shear rates. However, to obtain a profound understanding of polymer processing, there is a clear need for data at high shear rates and high shear strains. The shear cell used in this study enables us to access this region.

It is well established that isotactic PB-1, like many other polyolefins, is polymorphous, exhibiting different morphologies depending on thermal treatment and mechanical handling. At least five different crystal modifications have been reported in the literature: ${ }^{31-34}$ two hexagonal ones, Form I and Form I', two tetragonal ones, Form II and Form II', and an orthorhombic one, Form III. Cooling from the melt at atmospheric pressure results in the growth of unstable crystals of Form II which then transform into Form I crystals. This transformation takes place during days or weeks at room temperature and significantly changes the properties of PB-1 mainly through a change in density (the unstable Form II is transparent, soft, and easily deformed, whereas the stable Form I is translucent, rigid, and not prone to mechanical deformation). Forms I' and II' are obtained when the material is crystallized under high pressure, and Form III has been observed in the case of crystallization from solution. In this paper the influence of processing parameters on this crystal transition (Form II to Form I) will be briefly highlighted.

\section{Experimental Section}

Materials. The two isotactic PB-1s (PB0400 and PB0800) used in this study are commercial grades provided by Basell Polyolefins in the form of pellets. Both grades have an isotacticity of $98.8 \%$ and contain no nucleating agents. PB0400 has a $M_{\mathrm{w}}=176 \mathrm{~kg} / \mathrm{mol}$ and a polydispersity of 5.7 ; PB0800 has a $M_{\mathrm{w}}=133 \mathrm{~kg} / \mathrm{mol}$ and a polydispersity of 4.3 (data obtained from GPC measurements by Basell Polyolefins). More material properties and a rheological characterization can be found in the Supporting Information.

Methods. For the experiments, a shear cell ${ }^{27}$ developed at the Solvay Central Laboratory was used. It is a small sandwich-type cell in which the sample is uniaxially sheared between two oppositely moving glass windows, driven by a servomotor. The glass windows of the cell are incorporated in sample holders which are placed in independently heated conditioning blocks. The typical thickness of a sheared sample is $50-100 \mu \mathrm{m}$. The shear cell can be operated from the low shear rate region up to rates of $1500 \mathrm{~s}^{-1}$. Deformations up to a few hundred shear units can be achieved, which is typically what is realized in the outer layers during injection moulding. The main advantage of this shear cell is that the probed structure is homogeneous, i.e., there is a constant shear rate throughout the entire sample, which is not the case for the extrusion die configurations ${ }^{2,13,21-26}$ that have been used up to now.

Windows and holes are provided in the equipment to view the flow-vorticity plane of the sheared sample. An optical train consisting of a modulated laser allows to follow the transmitted intensity $I_{\mathrm{dc}}$ and the birefringence $\Delta n^{\prime}$ during shear flow and subsequent crystallization. The transmitted intensity is normalized with respect to its initial value $I_{\mathrm{dc}}(t=0)$ and the birefringence $\Delta n^{\prime}$ is obtained from

$$
\Delta n^{\prime}=\frac{\lambda \delta^{\prime}}{2 \pi e}
$$

Here $e$ is the sample thickness, $\lambda$ the laser wavelength $(670 \mathrm{~nm})$, and $\delta^{\prime}$ the optical retardation calculated from

$$
\left|\sin \delta^{\prime}\right|=\frac{\sqrt{{I_{\mathrm{sin}}{ }^{2}+I_{\mathrm{cos}}^{2}}^{2}}}{I_{\mathrm{dc}}}
$$

with $I_{\sin }$ and $I_{\cos }$ the amplitudes of a particular sine and cosine component in the detected laser beam. ${ }^{27}$

To erase the thermal and flow history, the samples were always annealed at $200{ }^{\circ} \mathrm{C}$ for $10 \mathrm{~min}$ in a separate oven. Subsequently they were cooled to $150{ }^{\circ} \mathrm{C}$ and transferred to the conditioning blocks kept at the desired crystallization temperature $T_{\mathrm{c}}$. This procedure avoids long cooling times typical of the already mentioned extrusion die configurations..$^{2,13,21-26}$ The thermal characteristics of the setup resulted in a time of about $10 \mathrm{~min}$ to quench the sample to the measurement temperature. For isothermal measurements this leads to average cooling rates of $10{ }^{\circ} \mathrm{C} / \mathrm{min}$, comparable to the cooling characteristics of the Linkam cell. The measurements were carried out at three different crystallization temperatures $T_{\mathrm{c}}$, namely, 93,98 , and $103{ }^{\circ} \mathrm{C}$. The choice of the three test temperatures was dictated by two important constraints. On one hand, temperatures were chosen so as to determine measurable characteristic crystallization times. In fact, too high temperatures (close to the melting temperature $T_{\mathrm{m}}=109.6^{\circ} \mathrm{C}^{30}$ ) resulted in very low crystallization rates, whereas too low temperatures caused an essentially instantaneous crystallization. In both cases, the measurement of the crystallization time becomes impossible or at best unreliable. On the other hand, the three temperatures were chosen in order to determine the largest possible difference in crystallization time, to guarantee the highest amplification of the temperature effect.

For the experiments, zero time scale was assigned to the start of the shear flow, corresponding to the instant at which the crystallization temperature $T_{\mathrm{c}}$ was reached. Shear flow was applied with varying shear rates $\dot{\gamma}$ and shear strains $\gamma$. The shear rate $\dot{\gamma}$ and the shear strain $\gamma$ were calculated from the velocity $v$ and the displacement $s$ of the sample holders according to

$$
\dot{\gamma}=\frac{v}{e / 2} \text { and } \gamma=\frac{s}{e / 2}
$$

As with the calculation of the birefringence $\Delta n^{\prime}$ from eq 1 , these calculations require the thickness $e$ of the sheared sample. Here the thickness of the sample, recovered after solidification, was systematically used. This thickness could only be determined with an accuracy of a few micrometers, resulting in a relative error of about $5 \%$ for the birefringence, the shear rate, and the shear strain. To ensure that the shear profile was boxlike and that the set velocity $v$ and displacement $s$ were correctly imposed, the velocity profile was recorded using laser tracking of the position of one of the sample holders. This is particularly relevant near the limitations of the apparatus. More details on the construction, the temperature control and the limitations of the apparatus, the optical setup, and the calculation of the birefringence can be found in ref 27 .

\section{Results and Discussion}

Typical Curves and Characteristic Values. By use of the setup described in the previous section, several series of experiments were carried out varying flow parameters (shear rate $\dot{\gamma}$ and shear strain $\gamma$ ) and crystallization temperatures $T_{\mathrm{c}}$. In all the experiments the transmitted laser light was measured during shear flow and subsequent isothermal crystallization. In this first section the typical shapes of the obtained curves will be discussed and characteristic values will be defined.

Flow Birefringence. Flow birefringence is the term commonly used to indicate the development of birefringence during flow. In Figure 1 the flow birefringence of PB0400 is plotted for different shear rates $\dot{\gamma}\left(T_{\mathrm{c}}=93{ }^{\circ} \mathrm{C}\right.$ and $\left.\gamma=100\right)$. The instant at which the shear flow is stopped is indicated by circles. For not too low shear rates (the birefringence value has to be sufficiently large to be distinguishable from the noise level) the 


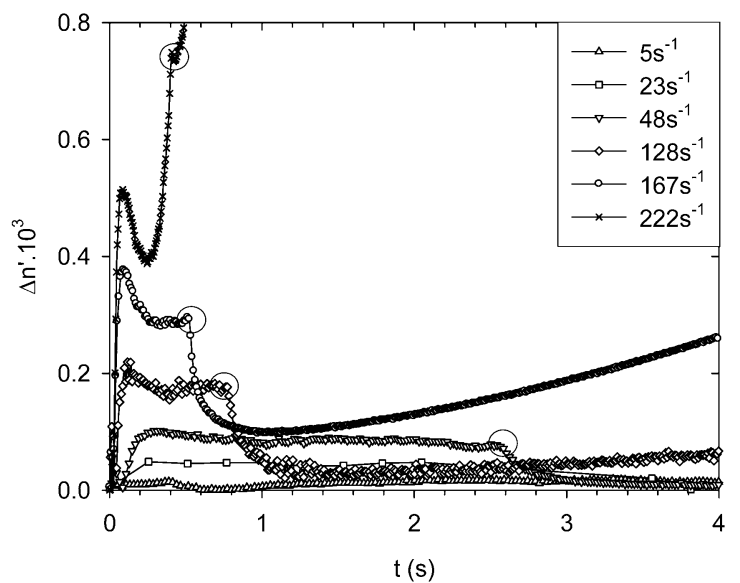

Figure 1. Flow birefringence of $\operatorname{PB} 0400\left(T_{\mathrm{c}}=93^{\circ} \mathrm{C}\right.$ and $\left.\gamma=100\right)$. Circles indicate the end of each shear pulse.

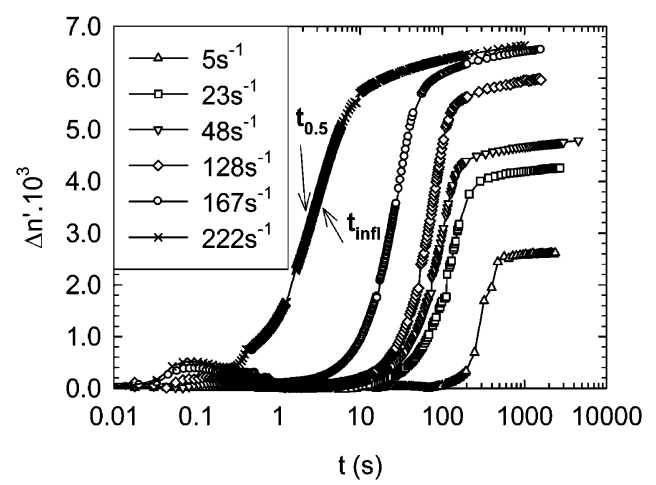

Figure 2. Birefringence development during isothermal crystallization of PB0400 after shearing with different shear rates $\left(T_{\mathrm{c}}=93{ }^{\circ} \mathrm{C}\right.$ and $\gamma$ $=100)$.

flow birefringence first shows a slight overshoot and then stabilizes to a plateau level. The observed overshoots on shear start-up have been rationalized in terms of the stretching of polymer chains in the melt, ${ }^{2}$ which leads to overshoots in the normal stress differences $N_{1}$ and $N_{3}$. The plateau value is related to a level of molecular orientation and can also be associated to the normal stresses as dictated by the stress optical rule. ${ }^{35}$ As can be seen in Figure 1, this plateau value increases with shear rate. After the shear pulse the flow stress, and therefore the flow birefringence, relaxes. However, for the highest shear rate, $\dot{\gamma}=222 \mathrm{~s}^{-1}$, there is an unusual upturn in the flow birefringence curve and the birefringence hardly relaxes after cessation of flow. In their extrusion die experiments, Kumuraswamy et al. ${ }^{2,25}$ also pointed out the presence of such an upturn above a critical shear stress and strain. This maximum was attributed to a change in the crystallization mechanism, i.e., the formation of a "shear-induced structure" related to the presence of oriented precursors. Even for $\dot{\gamma}=167 \mathrm{~s}^{-1}$, where there is no characteristic upturn in flow birefringence, the birefringence does not relax completely. This points toward a rather gradual transition from transient birefringence of melt flow to one that has a significant contribution due to oriented structures generated during shear.

Birefringence Development after Shear. The birefringence $\Delta n^{\prime}$ was monitored not only during shear flow (indication of molecular orientation) but also during the subsequent crystallization (indication of orientation/anisotropy development). Figure 2 shows the typical evolution of birefringence during isothermal crystallization of $\mathrm{PB} 0400\left(T_{\mathrm{c}}=93{ }^{\circ} \mathrm{C}\right.$ and $\left.\gamma=100\right)$. For quiescent crystallization experiments (not shown here) the birefringence remains at its initial value and does not increase

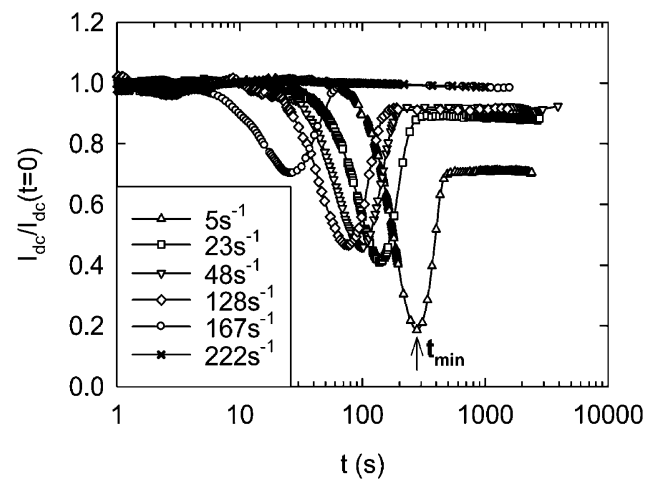

Figure 3. Transmitted intensity during isothermal crystallization of PB0400 after shearing with different shear rates $\left(T_{\mathrm{c}}=93^{\circ} \mathrm{C}\right.$ and $\gamma=$ 100).

in time. This is what one would expect for a spherulitic structure since the signal is averaged over all possible directions. For the lowest shear rate in Figure 2, $\dot{\gamma}=5 \mathrm{~s}^{-1}$, there is an increase in birefringence. This indicates that the applied shear flow resulted in the development of orientation or anisotropy in the crystallizing sample, although rather limited. With increasing shear rate the time needed for the crystalline orientation to become visible in the birefringence signal decreases and the maximum value of the birefringence increases. These are indications of the faster crystallization kinetics and the development of a more pronounced anisotropic structure, respectively. Although for the highest shear rate $\dot{\gamma}=222 \mathrm{~s}^{-1}$ the increase in birefringence is visible within a time span as short as $0.5 \mathrm{~s}$, much longer times are needed to complete the crystallization.

After the birefringence reached some kind of plateau level it can still display a very slow increase, caused by secondary crystallization. An interesting feature of the above curves is that with increasing shear rate there is a more pronounced secondary crystallization mechanism. This trend was also observed for an i-PP, ${ }^{27}$ and it was explained in the following way: at lower shear rates, where the structure is spherulitic, the secondary crystallization process mainly adds an isotropic component, having no affect on the birefringence, whereas at high shear rates the overall orientation is so dominant that any process that tends to improve the existing lamellae or adds lamellae by an insertion mechanism can only reinforce the orientation.

To characterize the crystallization kinetics the time needed to reach $50 \%$ of the maximum of the birefringence, defined as $t_{0.5}$, is used. This characteristic time is indicated in Figure 2 for $\dot{\gamma}=222 \mathrm{~s}^{-1}$.

Turbidity. After cessation of shear flow, the sample crystallizes isothermally and the intensity of transmitted light changes with time. Figure 3 shows the transmitted intensity, normalized with respect to its initial value, during isothermal crystallization of PB0400 $\left(T_{\mathrm{c}}=93{ }^{\circ} \mathrm{C}\right.$ and $\left.\gamma=100\right)$. Initially the intensity is constant. During this time the polymer remains essentially in the state of the undercooled melt. As crystallization sets in, the nucleation and subsequent growth of the crystallites generate a strong and relatively fast increase of the sample turbidity, which corresponds to a reduction of the intensity. At later stages the curves show a pronounced minimum of which the position and the magnitude depend on the applied shear rate. Spruiell and Ding ${ }^{36}$ attributed the presence of this minimum to impingement, since the impinging structures loose their optical contrast and hence their ability to scatter light. The minimum becomes less distinguishable at high shear rates, where there is almost no loss in intensity. Similar observations were made for an i-PP. ${ }^{27}$ The instant for which the intensity reaches its minimum, defined 


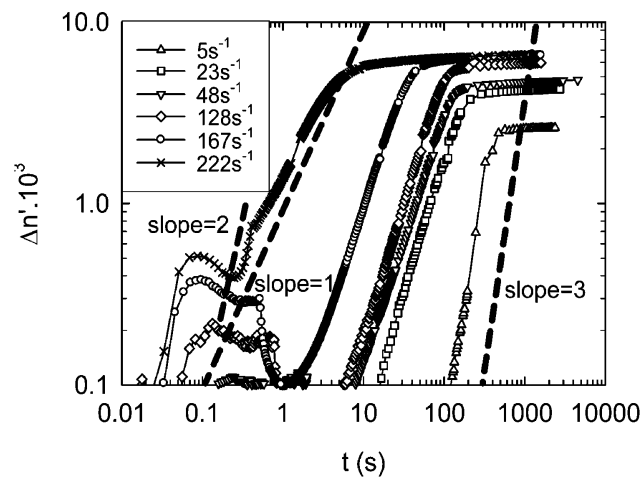

Figure 4. Birefringence development during isothermal crystallization of PB0400 after shearing with different shear rates $\left(T_{\mathrm{c}}=93{ }^{\circ} \mathrm{C}\right.$ and $\gamma$ $=100)$. Slopes are added for comparison.

as $t_{\min }$, was again used to qualitatively determine the crystallization kinetics. This characteristic time is indicated in Figure 3 for $\dot{\gamma}=5 \mathrm{~s}^{-1}$.

Growth Speed. Kumaraswamy et al. ${ }^{26}$ discussed the evolution of birefringence, as described in one of the previous sections, with respect to the crystal growth rate. For highly oriented cylindrical growth from a central shish, the birefringence is directly proportional to the volume of the growing structure. Therefore, a constant linear (i.e., radial) growth rate implies that the birefringence must increase as $t^{2}$. However, in their experiments this was only the case during flow. After cessation of flow, a linear growth for the birefringence was observed. They showed that this behavior of the birefringence could also be modeled by cylindrical growth assuming that the linear growth rate of the cylindrical crystal is higher during shear than after shear cessation. This assumption is in agreement with their wide-angle $\mathrm{X}$-ray diffraction data, where a change from a quadratic growth rate during flow to a linear growth rate after cessation of flow was observed. For the results presented here, a similar behavior is found. The slope of the birefringence curve (on a $\log -\log$ scale) for the highest shear rate $\dot{\gamma}=222 \mathrm{~s}^{-1}$ indeed changes from 2 during flow to 1 (even somewhat below) after cessation of shear. For lower shear rates, the change in slope is not relevant since there is no characteristic upturn in the flow birefringence signal indicative of cylindrical growth. In these cases the slope after cessation of shear is larger than 1 and increases with decreasing shear rate, reaching a value of 3 for the lowest shear rate $\dot{\gamma}=5 \mathrm{~s}^{-1}$. Figure 4 illustrates this behavior.

Characteristic Times. As discussed in the previous sections, both birefringence and turbidity measurements can be used as a characteristic time scale for the crystallization kinetics. In Figure $5 t_{0.5}$ and $t_{\min }$ of PB0400 are plotted as a function of the shear rate $\left(T_{\mathrm{c}}=93{ }^{\circ} \mathrm{C}\right.$ and $\left.\gamma=100\right)$. It can be seen that there is a good quantitative agreement between both crystallization times. On one hand the value of $t_{0.5}$ corresponds quite well with the point of inflection, $t_{\text {infl }}$, in the part of the curve displaying the birefringence development after shear. Near this point the curvature changes from convex to concave, indicative of a change in the crystalline growth rate. The inflection point is indicated in Figure 2 for $\dot{\gamma}=5 \mathrm{~s}^{-1}$. On the other hand the minimum in transmitted intensity can be interpreted as the point of impingement of the growing crystallites. ${ }^{36}$ Thus, since one expects some change in the growth rate with impingement of the crystalline structures this could explain the quantitative agreement between $t_{0.5}$ and $t_{\min }$. There is also an obvious change in the slope of the curve at higher shear rates, which seems to correspond to the characteristic upturn in flow birefringence (indicated by gray symbols).

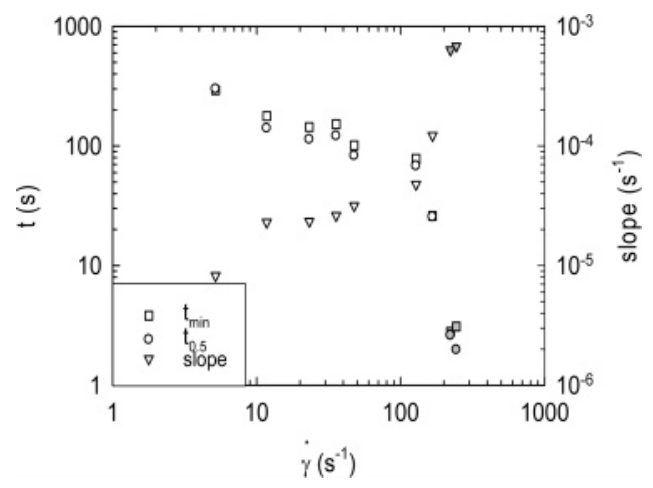

Figure 5. Characteristic crystallization times and slopes of the birefringence curves as a function of shear rate $\left(T_{\mathrm{c}}=93^{\circ} \mathrm{C}\right.$ and $\gamma=$ 100). The presence of the characteristic upturn in the flow birefringence is indicated by gray symbols.

The slopes of the birefringence curves near $t_{0.5}$ (calculated on a double linear scale) as a function of the shear rate are also plotted in Figure 5. The resulting curve displays a very similar but inverse behavior as the curves formed by the crystallization times. There is again an obvious change in the slope of the curve at higher shear rates, accompanying the unusual upturn in the flow birefringence (gray symbols).

For the samples subjected to shear rates up to $40 \mathrm{~s}^{-1}$ transcrystallinity was observed on optical micrographs of crosssections of the sheared samples. To investigate the effect of transcrystallinity on the measurements some of the experiments were repeated using different sample thicknesses. There was no observable effect of the gap width on the birefringence curves, indicating that the observed transcrystallinity does not influence the measured value of the birefringence. The minimum in the transmitted intensity reaches a lower value with increasing gap due to increased scattering but its position does not change. Thus, transcrystallinity does not affect the observed crystallization kinetics and the characteristic times can hence be used to probe possible scaling relations.

Temperature Effect. A parameter that strongly affects flowinduced crystallization is the crystallization temperature. In Figure 6 (top) $t_{\min }$ and $t_{0.5}$ of PB0400 as defined in the previous sections are plotted as a function of shear rate for three different crystallization temperatures $(\gamma=100)$. Looking at these curves, roughly three different regimes can be distinguished. This is illustrated by three straight lines for the data points obtained at a crystallization temperature $T_{\mathrm{c}}=93{ }^{\circ} \mathrm{C}$.

The first regime shows a decrease in $t_{\min }$ and $t_{0.5}$ with increasing shear rate. It is not very pronounced in Figure 6 (top), but from the many studies on flow-induced crystallization in the low shear rate region, ${ }^{1}$ i.e., $\dot{\gamma} \leq 10 \mathrm{~s}^{-1}$, one can easily imagine the presence of this first regime. The dotted lines in Figure 6 (top) indicate the quiescent crystallization times as obtained from a previous study on PB-1. ${ }^{12}$ These lines also represent the plateau at very low shear rates where flow has no effect on the crystallization kinetics. This plateau precedes the first regime, but with the setup used in this study it was not experimentally accessible. It also has to be mentioned that rheooptical measurements certainly are not the best way to determine the quiescent crystallization times. Because of the very low nucleation density of PB-1 under quiescent conditions it is very difficult to determine them accurately. In one experiment a spherulite can start growing immediately in the observed area, while in a rerun of the same experiment it can take much longer before a growing spherulite comes into the observation zone.

The second regime is a shear independent plateau. Such a plateau was already observed for iPPs by Vleeshouwers and 

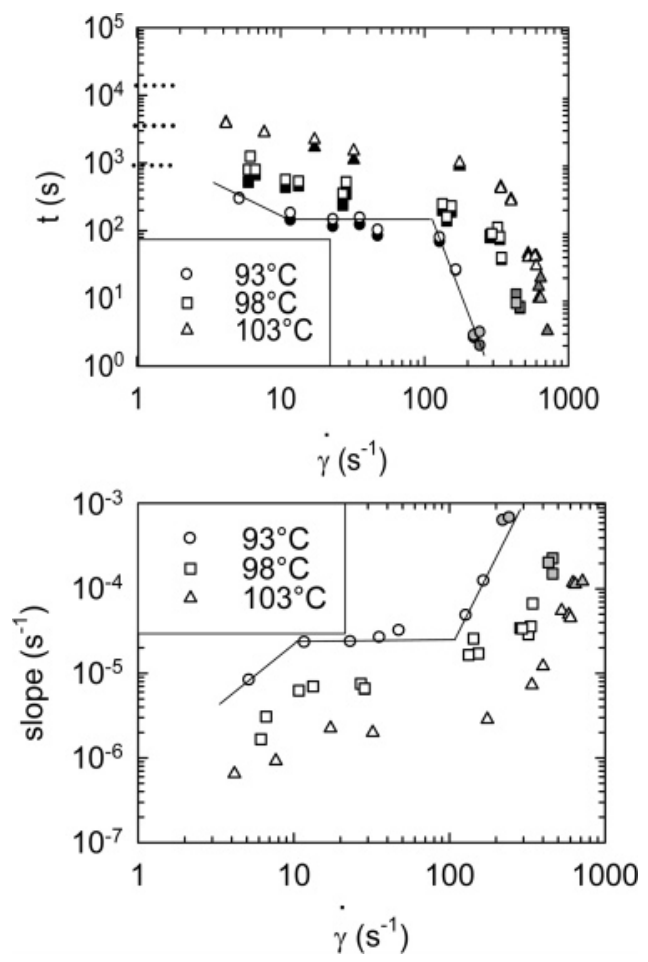

Figure 6. Crystallization times $t_{0.5}$ (filled symbols) and $t_{\min }$ (empty symbols) (top) and slopes of the birefringence curves (bottom) of PB0400 as a function of shear rate for different crystallization temperatures $(\gamma=100)$. The dotted lines represent the quiescent crystallization times. The presence of the characteristic upturn in the flow birefringence is indicated by gray symbols.

Meijer ${ }^{14}$ for the induction time as a function of shear rate, by Somani et al. ${ }^{19}$ for the half-time of crystallization as a function of shear rate and by Kumaraswamy et al. ${ }^{2}$ in plots of characteristic crystallization times as a function of shearing time. The latter authors suggested that this saturation could be due to the consumption of high- $M_{\mathrm{w}}$ chains in the creation of oriented structures. It should be noted however that for the data presented in Figure 6 the presence of a plateau is arguable, especially for the data at 98 and $103{ }^{\circ} \mathrm{C}$. Both our data on PB-1 and the data of Langouche ${ }^{27}$ on an iPP suggest the existence of a plateau level but are not at all conclusive. On the other hand, in the study of Vega et al. ${ }^{37}$ on a linear and a branched iPP the induction times do not display a plateau and show only two different regimes for the flow-induced crystallization. Moreover, Vleeshouwers and Meijer ${ }^{14}$ and Somani et al. ${ }^{19}$ did not observe a further increase in the crystallization kinetics with increasing shear rate after the plateau level. This is probably due to the fact that their experiments were limited to lower shear rates than those applied in this study.

Above a certain shear rate, depending on the crystallization temperature, the characteristic crystallization times again decrease with shear rate. This is the third regime. Here the characteristic times scale as $\dot{\gamma}^{-6}$ which is substantially different from the $\dot{\gamma}^{-2}$ behavior of $t_{\min }$ observed for an iPP. ${ }^{27}$

In Figure 6 (bottom) the slopes of the birefringence curves are plotted for the same three crystallization temperatures. The curves show a strikingly similar, although inverse, behavior as the curves formed by the characteristic crystallization times. Again the three different regimes can be distinguished and above a certain crystallization temperature-dependent shear rate the data points now scale as $\dot{\gamma}^{6}$. The presence of a shear independent plateau is again arguable.

In the following sections an attempt is made to link our results with the classification of flow-induced crystallization proposed by van Meerveld et al. ${ }^{38}$ For the rheological description it is important to distinguish two time scales. First, the reptation time, $\tau_{\text {rep }}$, associated with the reptation process and the orientation of the polymer chain and, second, the time scale $\tau_{\mathrm{s}}$ for the faster chain retraction and the stretch ratio $\lambda$. The stretch ratio is defined as the ratio of the current length of the contour path of the polymer chain, $L$, to the equilibrium value $L_{0}, \lambda=L / L_{0}$. For typical polymer melts the magnitude of $\tau_{\text {rep }}$ and $\tau_{\mathrm{s}}$ are separated by at least 2 orders of magnitude. This observation directly implies that the polymer chain can be oriented at much lower shear rates compared to those required to stretch it. This is conveniently expressed by two Deborah numbers, $D e$, based on $\tau_{\text {rep }}$ and $\tau_{\mathrm{s}}$, defined as

$$
\begin{gathered}
D e_{\text {rep }}=\tau_{\text {rep }} \dot{\gamma} \\
D e_{\mathrm{s}}=\tau_{\mathrm{s}} \dot{\gamma}
\end{gathered}
$$

with $\dot{\gamma}$ the shear rate. If the chain is stretched, two different regimes can be identified. For small $\lambda$, the chain maintains a Gaussian configuration and the amount of rotational isomerization is small. At large $\lambda$, the chain configuration becomes non-Gaussian and the amount of rotational isomerization is large. The parameter $\lambda^{*}$ denotes the transition between these two chain stretching regimes, which may be identified as weak and strong stretching conditions, respectively.

According to van Meerveld et al. ${ }^{38}$ the influence of flow can be separated into four regimes. For $D e_{\text {rep }}$ and $D e_{\mathrm{s}}<1$, the chains are at equilibrium. Subsequently three transitions are identified corresponding to increasing orientational order of the contour path with $D e_{\text {rep }}>1$, the onset of chain stretching with $D e_{\mathrm{S}}>$ 1 and finally the onset of rotational isomerization with $\lambda>$ $\lambda *(T)$. To characterize these flow regimes four different procedures were proposed, based on different ways to estimate the reptation and stretching times of the chains. ${ }^{38}$ An evaluation of experiments reported in the literature illustrates that the transition from an enhanced nucleation rate of spherulites toward the development of the shish-kebab structure correlates with the transition from the orientation of the chain segments to the rotational isomerization of the high- $M_{\mathrm{w}}$ chains in the melt. Similar results were obtained by Vega et al. ${ }^{37}$ who applied the classification to their results.

In this study the focus is on the point where the upturn in flow birefringence appears and the onset point of the regime that scales with the sixth power. As mentioned earlier these two points correspond rather well. One can imagine that these points correspond to the onset of strong chain stretching, i.e., $D e_{\mathrm{s}}>1$ and $\lambda>\lambda^{*}(T)$. According to van Meerveld et al..$^{38}$ it is most appropriate to use $\tau_{\text {rep }}{ }^{\mathrm{HMW}}$ and $\tau_{\mathrm{s}}{ }^{\mathrm{HMW}}$ based on the high$M_{\mathrm{w}}$ chains in the melt. Here $\tau_{\text {rep }}{ }^{\mathrm{HMW}}$ is derived from the point where the storage and loss moduli $G^{\prime}(\omega)$ and $G^{\prime \prime}(\omega)$ start to deviate from their limiting behavior at low frequencies. This specific method was not used by van Meerveld et al., ${ }^{38}$ but it is merely a different way to get an idea of the relaxation behavior of the high- $M_{\mathrm{w}}$ chains in the melt. $\tau_{\mathrm{s}}{ }^{\mathrm{HMW}}$ is then calculated as $\tau_{\mathrm{s}}^{\mathrm{HMW}}=\left(\tau_{\mathrm{rep}}{ }^{\mathrm{HMW}} / 3 Z\right)$. For the number of entanglements per chain $Z=Z_{\mathrm{w}}=\left(M_{\mathrm{w}} / M_{\mathrm{e}}\right)$ was used, with $M_{\mathrm{e}}=18000 \mathrm{~g} / \mathrm{mol}$ the entanglement molecular weight. ${ }^{39}$

The results of calculating the characteristic Deborah number corresponding with the transition to the highly oriented regime in flow-induced crystallization of PB0400 are summarized in Table 1. For the procedure used to characterize the flow regimes it seems that the values of $D e_{\mathrm{s}}$ are identical (within 2\%) for the data at 103 and $98{ }^{\circ} \mathrm{C}$. For the data at $93{ }^{\circ} \mathrm{C}$ however there is a difference of about $50 \%$. The reason for this difference is not 
Table 1. Characteristic Relaxation Times of the High- $M_{\mathrm{w}}$ Tail of PB0400, Corresponding Stretching Times, and Critical Values of the Deborah Number Corresponding with the Sharp Increase in Crystallization Kinetics

\begin{tabular}{rccc}
\hline$T\left({ }^{\circ} \mathrm{C}\right)$ & $\tau_{\text {rep }}{ }^{\mathrm{HMW}}$ & $\tau_{\mathrm{s}}{ }^{\mathrm{HMW}}$ & $D e_{\mathrm{s}}(-)$ \\
\hline 93 & 4.92 & 0.17 & 19 \\
98 & 3.99 & 0.14 & 35 \\
103 & 3.26 & 0.11 & 34
\end{tabular}

yet clear, but it could be due to the fact that $\lambda^{*}=\lambda *(T)$ and decreases with decreasing temperature. ${ }^{38}$ The values for $D e_{\mathrm{s}}$ correspond rather well with the values presented by van Meerveld et al. ${ }^{38}$ and the short time shear results of Vega et al. ${ }^{37}$ They calculated values for $D e_{\mathrm{s}}=0.5-80$ and $D e_{\mathrm{s}}=20$, respectively, when applying a similar method for the determination of the characteristic relaxation time, i.e., estimating $\tau_{\text {rep }}{ }^{\mathrm{HMW}}$ from the longest time in the relaxation spectrum.

Strain Effect. Not only the shear rate and the crystallization temperature are of importance in flow-induced crystallization experiments. The total shear strain $\gamma=\dot{\gamma} t_{\mathrm{s}}$ (which for a constant shear rate is equivalent to the shearing time) also affects the polymer orientation and thus the crystallization kinetics. This was already observed for low shear rates $\left(\dot{\gamma} \leq 5 \mathrm{~s}^{-1}\right)$ by Bove and Nobile ${ }^{3}$ who stated that both shear rate and shear strain are critical factors to form stable nuclei for the crystallization process from the flow oriented polymer chains. Figure 7 shows the flow birefringence of PB0400 for experiments carried out at the same crystallization temperature $T_{\mathrm{c}}=103{ }^{\circ} \mathrm{C}$ and a fixed shear rate $\dot{\gamma}=620 \mathrm{~s}^{-1}$, only varying the shear strain. For the lowest strains $(\gamma=36$ and 55) the flow birefringence relaxes completely despite of the high shear rate that was applied. For the intermediate strain $(\gamma=73)$ an incomplete relaxation is observed, and for even higher strains $(\gamma=109$ and $\gamma=145)$ this incomplete relaxation is accompanied by a characteristic upturn in the flow birefringence. From Figure 7 it can thus be concluded that a high shear rate only is not enough to attain the transition to shear-induced structures. The shear rate also has to be applied for a sufficiently long time. In other words: a critical shear strain exists. This is also clear in the classification of van Meerveld which contains two requirements for the onset of strong chain stretching: $D e_{\mathrm{s}}>1$ (related to a critical shear rate) and $\lambda>\lambda *(T)$ (related to a critical shear strain).

In experiments with an extrusion die Janeschitz-Kriegl and co-workers $^{13,23,24}$ showed, based on micrographs of cross sections of the extracted samples, that the boundary between the highly oriented layer and the fine-grained layer is characterized by $\dot{\gamma}^{4} t_{s}{ }^{2}=$ const. if the relaxation time of the threadlike precursors $\tau_{1}$ is large compared to $t_{\mathrm{s}}$ and by $\dot{\gamma}^{4} t_{\mathrm{s}}=$ const. if $\tau_{1}$ is small compared to $t_{\mathrm{s}}$. They proposed a nucleation model assuming that threadlike precursors start growing from spotlike nuclei which are not present from the beginning but are formed sporadically during the shear treatment. By assumption of a fixed value for the total length of precursors per unit volume at the boundary of the highly oriented layer depending on the magnitude of $t_{\mathrm{s}}$ relative to the magnitude of the relaxation time of the threadlike precursors, this resulted in a correct prediction of the observed behavior. Although there are a few crystallization models that include shearing time as a parameter, for example, the models of Schneider et al. ${ }^{40}$ and Zuidema et al. ${ }^{41}$ (a modification of Schneider's model), most of the existing models do not take into account the shearing time. As could be seen from Figure 7 and as will be shown in the rest of this section, this is a serious shortcoming. Just like parameters containing the material behavior, shearing time also is a parameter which cannot be disregarded in a realistic model of flow-induced crystallization.

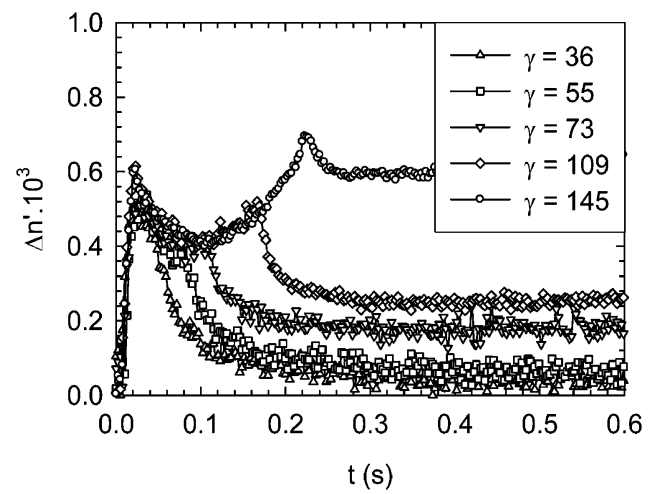

Figure 7. Flow birefringence of $\mathrm{PB} 0400$ for experiments carried out at the same crystallization temperature $\left(T_{\mathrm{c}}=103^{\circ} \mathrm{C}\right)$ and shear rate $(\dot{\gamma}$ $=620 \mathrm{~s}^{-1}$ ), only varying shear strain

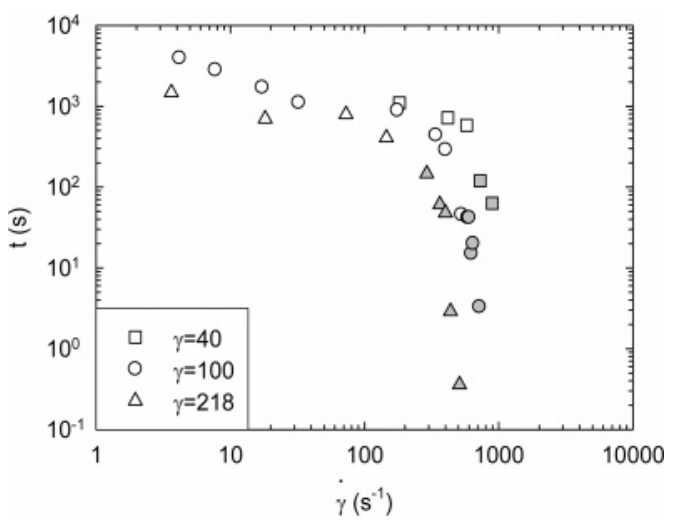

Figure 8. Crystallization time $t_{0.5}$ of PB0400 as a function of shear rate for different shear strains $\left(T_{\mathrm{c}}=103{ }^{\circ} \mathrm{C}\right)$. The presence of the characteristic upturn in the flow birefringence is indicated by gray symbols.

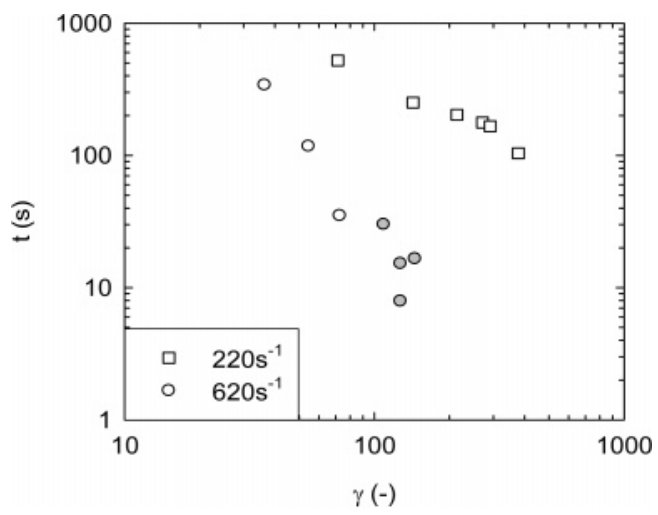

Figure 9. Crystallization time $t_{0.5}$ of PB0400 as a function of shear rates for different shear strains $\left(T_{\mathrm{c}}=103^{\circ} \mathrm{C}\right)$.

In Figure 8 the characteristic crystallization time $t_{0.5}$ of PB0400 is plotted vs shear rate for different shear strains. $t_{\text {min }}$ is not plotted here for clarity and because this characteristic time is difficult to determine for high shear rates where almost no loss in intensity is observed. As expected, increasing the shear strain enhances the crystallization kinetics, indicated by the shorter crystallization times. Also, with increasing shear strain the transition to shear-induced structures, indicated by gray symbols, occurs at lower shear rates. A plot of the slopes of the birefringence curves (not presented) again shows a very similar but inverse behavior.

In Figure 9 the characteristic crystallization time $t_{0.5}$ is plotted as a function of shear strain for two different shear rates. For the lowest shear rate $\dot{\gamma}=220 \mathrm{~s}^{-1}$ the transition to shear-induced structures, characterized by a sharp decrease in crystallization 


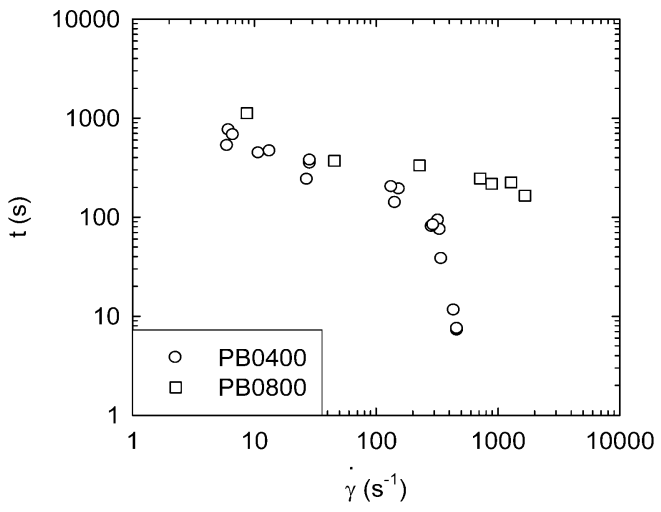

Figure 10. Crystallization time $t_{0.5}$ as a function of shear rate for two samples with different $M_{\mathrm{w}}\left(T_{\mathrm{c}}=98^{\circ} \mathrm{C}\right.$ and $\left.\gamma=100\right)$.

time, could not be attained within the experimental limits of the shear cell. For a shear rate $\dot{\gamma}=620 \mathrm{~s}^{-1}$, however, this transition is observed, again corresponding to the characteristic upturn in the flow birefringence (gray symbols). From Figure 9 it can be seen that the critical shear strain necessary to attain a transition to shear-induced structures decreases with increasing shear rate and that, for a larger shear rate, shear strain has a more pronounced influence on crystallization kinetics as indicated by the steeper slope of the curve formed by the data points at $\dot{\gamma}=620 \mathrm{~s}^{-1}$.

Figures 8 and 9 confirm that shear strain is an important parameter that should be taken into account when developing new crystallization models.

Effect of Molecular Weight. The molecular weight also plays an important role in flow-induced crystallization of polymers since the length of the polymer chains largely determines their relaxation times and their orientation states during shear. This molecular orientation mediates the pathway to nucleation. In Figure 10 the crystallization time $t_{0.5}$ is plotted as a function of shear rate for both PB0400 and PB0800. Whereas the curve for PB0400 shows a sharp increase in crystallization kinetics at high shear rates, related to the presence of oriented precursors, this is not the case for the low molecular weight PB0800. The relaxation time of these chains is too short to form threadlike precursors, even for the highest applicable shear rate of $1500 \mathrm{~s}^{-1}$. According to Kumuraswamy et al. ${ }^{2,25}$ this is because the applied shear stress and/or strain are below their critical values needed to induce highly oriented crystallite growth in the sample. In reference to the classification made by van Meerveld et al. ${ }^{38}$ (as discussed in a previous section), this means that the corresponding Deborah numbers do not attain the value required for the onset of strong chain stretching, i.e., the fourth regime. It is expected that with increasing shear rate a sharp acceleration of the crystallization will eventually occur even for the low molecular weight sample. It is assumed that PB0800 will display this characteristic upturn for the same value of the Deborah number as is the case for PB0400, i.e., $D e_{\mathrm{s}} \approx$ 35. Since both polymers were investigated under the same conditions $\left(T_{\mathrm{c}}=98^{\circ} \mathrm{C}\right.$ and $\left.\gamma=100\right)$ and have a similar MWD, the assumption that $\lambda>\lambda^{*}(T)$ can be related to a single universal magnitude for $D e_{\mathrm{s}}$ is not so unreasonable. ${ }^{38}$ This results in a critical shear rate for PB0800 of approximately $1800 \mathrm{~s}^{-1}$, and from Figure 10 it can be seen that this is a plausible value. However, this could not be checked experimentally since this shear rate is beyond the limit of the shearing cell.

Morphology Change. As mentioned in the Introduction isotactic PB-1 exhibits different morphologies depending on thermal treatment and mechanical handling. The morphological transition that is most relevant in polymer processing operations

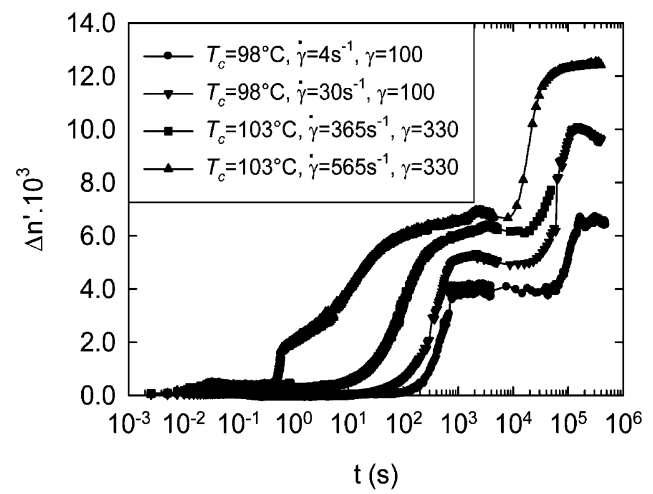

Figure 11. Birefringence curves for PB0400 under various conditions. The further upturn after cooling to room temperature indicates the Form II $\rightarrow$ I transformation.

is that of unstable crystals of Form II into Form I crystals. This transformation takes place during days or weeks at room temperature and significantly changes the properties of PB-1. ${ }^{31,32}$ It has been suggested in the literature ${ }^{42}$ that processing influences the rate of this transition. Here the influence of processing parameters on this crystal transition is briefly discussed based on some remarkable observations.

After the birefringence reached a plateau level, indicative of the completion of the primary crystallization process, some of the samples were kept in the shear cell and cooled to room temperature by switching off the oil baths of the conditioning blocks. In all cases a further upturn in birefringence appeared after some time, reflecting the Form II $\rightarrow$ I transformation. From Figure 11 it can be seen that the time between the start of the crystallization experiment and this characteristic upturn is of the order of $10^{4-} 10^{5} \mathrm{~s}$, which is smaller than the reported value ${ }^{31}$ for this transformation after crystallization under quiescent conditions. Figure 11 also shows that with increasing shear rate the time prior to the start of the transformation decreases, whereas the slope of the curves during this second upturn, being an indication of the transformation rate, increases with increasing shear rate. The latter observation is in accordance with previous findings ${ }^{42}$ that the transformation rate is increased by stress deformation. From these results it can thus be concluded that shear deformation is indeed effective in accelerating the Form II $\rightarrow$ I transformation.

\section{Conclusions}

The use of a new sandwich-type shear cell which can be operated up to shear rates of $1500 \mathrm{~s}^{-1}$ in combination with a modulated laser made it possible to present a global view of flow-induced orientation in two isotactic PB-1 samples with different $M_{\mathrm{w}}$. For varying levels of shear rate and shear strain and for several crystallization temperatures the transmitted intensity and birefringence values are presented throughout the complete crystallization process. At low shear rates the results show a gradual rise of anisotropy in the samples. At higher shear rates, this is followed by a much sharper rise in birefringence accompanied by a characteristic upturn in the birefringence pattern during flow, typical of the formation of shear-induced structures. The evolution of transmitted intensity shows a characteristic minimum for which both position and amplitude depend on the shearing conditions. On the basis of birefringence and transmitted intensity respectively, two characteristic crystallization times are defined between which a good quantitative agreement is found. The slope of the birefringence curves near $t_{0.5}$ increases with increasing shear rate and displays a strikingly similar but inverse behavior as the crystallization times. Curves 
for these three characteristic values as a function of shear rate or shear strain display a sharp change in slope occurring nearly together with the characteristic upturn in the flow birefringence pattern. It is shown that the sharp change in slope occurs at a fixed value of $D e_{\mathrm{s}}$ for the experiments at 103 and $98^{\circ} \mathrm{C}$. The calculated values are in accordance with results from previous studies on iPP. Results also confirm that the Fase II to Fase I transformation for PB-1 is enhanced by stress deformation as can be seen from a further upturn in the birefringence signal after cooling to room temperature.

Acknowledgment. The authors thank Dr. H. M. Laun from BASF and Dr. G. Jackers and J. De Clippeleir from Basell Polyolefins for providing the iPB samples. We are grateful to Solvay for allowing us to use their facilities at the Solvay Central Laboratory. Also, we thank G. W. M. Peters for the helpful discussions.

Supporting Information Available: A table containing the material properties and a figure showing the rheological behavior of PB0400 and PB0800. This material is available free of charge via the Internet at http://pubs.acs.org.

\section{References and Notes}

(1) Kumaraswamy, G. J. Macromol. Sci. Polym. Rev. 2005, 45, 375397.

(2) Kumaraswamy, G.; Issaian, A.; Kornfield, J. A. Macromolecules 1999, 32, 7537-7547.

(3) Bove, L.; Nobile, M. R. Macromol. Symp. 2002, 185, 135-147.

(4) Wolkowicz, M. D. J. Polym. Sci. 1978, 63, 365-382.

(5) Tribout, C.; Monasse, B.; Haudin, J. M. Colloid Polym. Sci. 1996, 274, 197-208.

(6) Jay, F.; Haudin, J. M.; Monasse, B. J. Mater. Sci. 1999, 34, 20892102 .

(7) Koscher, E.; Fulchiron, R. Polymer 2002, 43, 6931-6942.

(8) Acierno, S.; Palomba, B.; Winter, H. H.; Grizzuti, N. Rheol. Acta 2003, $42,243-250$.

(9) Elmoumni, A.; Winter, H. H.; Waddon, A. J. Macromolecules 2003, $36,6453-6461$.

(10) Janeschitz-Kriegl, H.; Ratajski, E.; Stadlbauer, M. Rheol. Acta 2003, 42, 355-364.

(11) Watanabe, K.; Takahashi, T.; Takimoto, J.; Koyama, K. J. Macromol. Sci. Phys. 2003, B42, 1111-1124.

(12) Baert, J.; Van Puyvelde, P. Polymer 2006, 47, 5871-5879.

(13) Liedauer, S.; Eder, G.; Janeschitz-Kriegl, H.; Jerschow, P.; Geymayer, W.; Ingolic, E. Int. Polym. Proc. 1993, 8, 236-244.

(14) Vleeshouwers, S.; Meijer, H. E. H. Rheol. Acta 1996, 35, 391-399.
(15) Pogodina, N. V.; Winter, H. H.; Srinivas, S. J. Polym. Sci., Polym. Phys. 1999, 37, 3512-3519.

(16) Pogodina, N. V.; Lavrenko, V. P.; Srinivas, S.; Winter, H. H. Polymer 2001, 42, 9031-9043.

(17) Somani, R. H.; Yang, L.; Hsiao, B. S.; Fruitwala, H. J. Macromol. Sci. Phys. 2003, B42, 515-531.

(18) Somani, R. H.; Yang, L.; Zhu, L.; Hsiao, B. S. Polymer 2005, 46, $8587-8623$

(19) Somani, R. H.; Hsiao, B. S.; Nogales, A.; Srinivas, S.; Tsou, A. H.; Sics, I.; Balta-Calleja, F. J.; Ezquerra, T. A. Macromolecules 2000, 33, 9385-9394.

(20) Agarwal, P. K.; Somani, R. H.; Weng, W.; Mehta, A.; Yang, L.; Ran, S.; Liu, L.; Hsiao, B. S. Macromolecules 2003, 36, 5226-5235.

(21) Eder, G.; Janeschitz-Kriegl, H.; Krobath, G. Colloid Polym. Sci. 1989, $80,1-7$.

(22) Liedauer, S.; Eder, G.; Janeschitz-Kriegl, H. Int. Polym. Proc. 1995, 3, 243-250.

(23) Jerschow, P.; Janeschitz-Kriegl, H. Int. Polym. Proc. 1997, 12, 7277.

(24) Eder, G.; Janeschitz-Kriegl, H. In Mater. Sci. Technol.; Meijer H. E. H., Eds.; Wiley-VCH: New York, 1997; Vol. 18; Chapter 5.

(25) Kumaraswamy, G.; Verma, R. K.; Issaian, A.; Wang, P.; Kornfield, J. A.; Yeh, F.; Hsiao, B. S.; Olley, R. H. Polymer 2000, 41, 89318940.

(26) Kumaraswamy, G.; Verma, R. K.; Kornfield, J. A.; Yeh, F.; Hsiao, B. S. Macromolecules 2004, 37, 9005-9017.

(27) Langouche, F. Macromolecules 2006, 39, 2568-2573.

(28) Coppola, S.; Grizutti, N.; Maffettone, P. L. Macromolecules 2001 34, 5030-5036.

(29) Acierno, S.; Grizutti, N.; Winter, H. H. Macromolecules 2002, 35, 5043-5048

(30) Hadinata, C.; Gabriel, C.; Ruellman, M.; Laun, H. M. J. Rheol. 2005, 49, 327-349.

(31) Danusso, F.; Giannotti, G. Makromol. Chem. 1965, 88, 149-158.

(32) Danusso, F.; Giannotti, G.; Polizzotti, G. Makromol. Chem. 1964, 80, 13-21.

(33) Nakafuku, C.; Miyaki, T. Polymer 1983, 24, 141-148.

(34) Kaszonyiova, M.; Rybnikar, F.; Geil, P. H. J. Macromol. Sci. Phys. 2004, B43, 1095-1114.

(35) Janeschitz-Kriegl, H. Polymer melt rheology and flow birefringence; Springer-Verlag: Berlin, 1983.

(36) Ding, Z.; Spruiell, J. E. J. Polym. Sci., Part B 1996, 34, 2783-2804.

(37) Vega, J. F.; Hristova, D. G.; Peters, G. W. M. Submitted.

(38) van Meerveld, J.; Peters, G. W. M.; Hütter, M. Rheol. Acta 2004, 44, 119-134.

(39) Acierno, S. Personal communication, 2006.

(40) Schneider, W.; Köppl, A.; Berger, J. Int. Polym. Proc. 1988, 2, $151-$ 180.

(41) Zuidema, H.; Peters, G. W. M.; Meijer, H. E. H. Macromol. Theory Simul. 2001, 10, 447-260.

(42) Armeniades, C. D.; Baer, E. J. Macromol. Sci. Phys. 1967, B1, 309. MA062068Q 